\title{
EL LÍMITE RACIONAL DE LA ESPECULACIÓN CIENTÍFICA EN LA COSMOLOGÍA CONTEMPORÁNEA, UNA CRÍTICA DESDE EL PENSAMIENTO DE NICHOLAS RESCHER
}

\author{
THE RATIONAL LIMIT OF SCIENTIFIC SPECULATION IN \\ CONTEMPORARY COSMOLOGY, A CRITIQUE FROM THE \\ NICHOLAS RESCHER'S THOUGHT
}

\author{
David Mayo Sánchez ${ }^{1}$ \\ Universidad de Salamanca (España)
}

Recibido: 28-2-2015

Aceptado: 18-5-2015

Resumen: La cosmología física contemporánea en su indagación acerca de la estructura del Universo ha llegado a formular hipótesis que postulan entidades no observables empíricamente y, ciertamente, sin posibilidad alguna de ser contrastadas; tal es el caso de la hipótesis cosmológica del multiverso. Esta hipótesis es uno de los casos más representativos de cosmología física especulativa, y, a la vez, es considerada por una gran parte de la comunidad científica como una teoría científica que podría explicar el origen del Universo y dar razón de sus leyes y constantes. Siendo así, parece ser que la ciencia es una empresa que lo puede explicar todo, a la vez que ha ocupado el lugar especulativo de la filosofía en su intento de dilucidar la realidad a nivel general y último. Desde la filosofía de Nicholas Rescher, semejante condición para la ciencia no es posible, ya que ésta no es perfecta y tiene límites. Para Rescher, la racionalidad científica está determinada por elementos epistemológicos y ontológicos, los cuales demarcan su cientificidad y sus alcances.

Palabras clave: cosmología especulativa; racionalidad científica; explicación; metafísica; límites de la ciencia.

\begin{abstract}
Contemporary physical cosmology in its inquiry into the structure of the universe has formulated hypotheses that posit unobservable empirically entities, which certainly have no possibility of being tested; such is the case of the multiverse cosmological hypothesis. This hypothesis is one of the most representative cases of speculative physical cosmology, and at the same time,
\end{abstract}

[1] (damayo1@gmail.com) Vinculado al al área de Lógica y Filosofía de la Ciencia del Departamento de Filosofía de la Universidad de Salamanca. 
is regarded by much of the scientific community as a scientific theory that could explain the origin of the universe and give an account of its laws and constants. Thus, it appears that science is an enterprise that can explain everything, while it has occupied the place of speculative philosophy in its attempt to elucidate reality to a general and ultimate level. From Nicholas Rescher's philosophy such a condition for science is not possible, because science is not perfect and has limits. For Rescher, scientific rationality is determined by epistemological and ontological elements which demarcate its scientific character and scope.

Keywords: speculative cosmology; scientific rationality; explanation; metaphysics; limits of science.

\section{Introducción}

La proliferación actual de ciertas especulaciones dentro del ámbito de la ciencia advierte acerca de una tendencia preponderante que está direccionando, concretamente al quehacer científico-cosmológico, hacia nuevos e inciertos terrenos epistemológicos y metafísicos; tal es el caso de la hipótesis cosmológica del multiverso ${ }^{2}$. La idea de un multiverso plantea la posibilidad de una realidad compuesta por un conjunto ilimitado de universos desconectados entre sí física y causalmente y, por lo tanto, «no podemos comunicarnos con ellos, ni podemos recibir ningún tipo señales físicas de ellos. En otras palabras, no podemos establecer su existencia por medios empíricos directos» ${ }^{3}$; y esto, en consecuencia, ha dado lugar a una serie de controversias generadas en el marco de la condición de racionalidad de tales hipótesis. Los debates dentro y fuera de la comunidad científica se han suscitado a partir de la crítica a la metodología científica utilizada en la elaboración de dichas hipótesis y, por ende, a sus conteni-

[2] En cuanto a la creciente producción intelectual relacionada a la cosmología física especulativa, el físico y cosmólogo alemán Hubert Goenner en un artículo publicado en 2010 titulado What kind of science is cosmology?, señala: «las partes especulativas [de la cosmología], que necesariamente implican la teorización física, son dominantes; solamente que ellas están comenzando a ser vinculadas a corroboraciones empíricas o todavía esperan a ser probadas en el futuro»; una cosmología que el mismo Goenner denomina como cosmología fantasía. Así, la cosmología especulativa se ha convertido en una rama de la ciencia física que ha roto con ciertos parámetros fundamentales de la metodología científica comúnmente aceptada; al mismo tiempo que — como indica Goenner- su presencia en el mundo académico-especializado y divulgativo es prevaleciente, sus producciones tanto en artículos, libros y actividades de investigación son abundantes. En Goenner, H.: «What kind of science is cosmology?» Annalen der Physik (Leipzig) 522, Nr. 6, 389-418 (2010); arXiv: astro-phy/2010.5532. pp. 1-62.

[3] Kragh, H.: Higher Speculations: Grand Theories and Failed Revolutions in Physics and Cosmology. New York: Oxford University Press, 2011, p. 260.

Thémata. Revista de Filosofía Nº53 (2016) pp.: 161-178. 
dos mismos, lo que ha llevado, en último término, a preguntar por la razón de ser de tales modelos cosmológicos.

Algunos científicos han señalado que las hipótesis cosmológicas del multiverso se han elaborado con el fin de dar una solución o explicación a problemáticas asociadas a la realidad tomada en su totalidad como lo son, por ejemplo, el origen del Universo o el ajuste fino de las leyes y constantes que le rigen. Estas indagaciones son las que históricamente han pertenecido a la especulación metafísica, que es la que desde la antigüedad ha tratado con la cuestión de la causalidad última y los primeros principios de la totalidad de lo real. Es por ello que la hipótesis del multiverso ha sido denunciada y situada en niveles filosóficos e incluso teológicos, bajo el argumento de que la razón fundamental del surgimiento de semejantes especulaciones está lejos de ser la de ofrecer un producto objetivo y consecuente de la teorización científica, sino que más bien, lo que ha impulsado a sus proponentes ha sido la intención de suplantar o competir con las opciones metafísicas tradicionales.

Las críticas hechas al multiverso están dirigidas a las diferentes versiones que han sido identificadas y clasificadas por el físico, teórico de cuerdas y también defensor de la misma hipótesis Brian Greene ${ }^{4}$. Dentro de la clasificación hecha por Greene se nombra a tres de ellos como: el multiverso inflacionario, el multiverso brana-cíclico y el multiverso final-matemático. Estos tipos de multiverso son los que más claramente manifiestan una propuesta metafísica.

Sin entrar en los detalles de cada uno de ellos, es de seguro que estos modelos cosmológicos incluyen una cierta base teórico-matemática, sin embargo, sus fines, reflejados en sus conclusiones, están relacionados a las implicaciones filosóficas anteriormente señaladas. Tal es el caso, por ejemplo, del cosmólogo de origen ruso Andrei Linde, que desde el escenario inflacionario afirma:

Dada la elección entre diferentes universos en esta estructura multiverso, podemos proceder mediante la eliminación de los universos donde sería imposible nuestra vida. Este paso simple es suficiente para la comprensión de las muchas características de nuestro Universo, que de otro modo podrían parecer milagrosas. ${ }^{5}$

De la misma manera, el físico teórico de la Universidad de Princeton y creador del modelo del multiverso brana-cíclico, Paul Steinhardt, y desde

[4] Brian Green, clasifica y denomina las diferentes propuestas del multiverso de la siguiente manera: multiverso mosaico, inflacionario, brana-cíclico, paisaje, cuántico, holográfico, simulado y final. En Green, B.: La realidad oculta. Universos paralelos y las profundas leyes del cosmos. Barcelona: Crítica, 2011, pp. 407-408.

[5] Linde, A.: «Infation, quantum cosmology, and the anthropic principle», en Barrow, J., P.

Thémata. Revista de Filosofía N53 (2016) pp.: 161-178. 
el contexto de teoría de cuerdas, así afirma explicando el modelo del universo Ekpirótico:

El modelo [Ekpirótico] se basa en la idea de que nuestro Universo Big Bang caliente fue creado a partir de la colisión de dos mundos tridimensionales en movimiento a lo largo de una dimensión extra oculta. Los dos mundos tridimensionales chocan y 'se adhieren', la energía cinética en la colisión se convierte en quarks, electrones, protones, etc., que se limitan a moverse a lo largo de tres dimensiones. La temperatura resultante es finita, por lo que la fase del Big Bang caliente comienza sin una singularidad. ${ }^{6}$

Por último, el físico y cosmólogo del Instituto Tecnológico de Massachusetts, Max Tegmark, postula lo que Green ha llamado el multiverso final, mismo que representa la más osada de todas las especulaciones, ya que en este modelo todas las posibilidades matemáticas son realizadas en diferentes niveles de multiversos. Así asevera Tegmark:

El multiverso Nivel IV, es denominado la 'teoría de Ensamble última' ya que $s u b$ sume todos los otros conjuntos, por lo tanto, trae cierre a la jerarquía de los multiversos, [...] Mientras que los universos de Nivel I se unen a la perfección, hay demarcaciones claras entre aquellos dentro de los niveles II y III causados por la inflación del espacio y la decoherencia, respectivamente. Los universos de Nivel IV son totalmente independientes y deben ser considerados en conjunto sólo para predecir. ${ }^{7}$

Evidentemente, todas estas declaraciones contienen serias implicaciones epistemológicas y ontológicas, que por su parte ponen en duda la validez racional de estas propuestas realizadas desde un contexto científico, a tal punto de crear una división en la comunidad de físicos y cosmólogos. Pero según los defensores de estas hipótesis —que representan la mayoría en cuanto a la producción intelectual - la ciencia podría estar encaminada a ofrecer las respuestas a las interrogantes últimas que por mucho tiempo han estado fuera de su competencia y, con ello, la ciencia pasaría, asegura Green, de los detalles a hacer algo más y eso «nos reta a reexaminar nuestra visión de la propia ciencia» ${ }^{8}$. Por su parte, el físico, premio Nobel y partidario del multiverso Steven Wienberg, asegura que «los científicos del futuro nos envidiarán un poco a los físicos de hoy porque estamos aún

Davies, P., Harper, C.: (eds.) Science and Ultimate Reality; quantum theory, cosmology, and complexity. Cambridge: Cambridge University Press, 2004, p. 441. (cursivas nuestras)

[6] Steinhardt,P.J. [en línea]: A Brief Introduction to the Ekpyrotic Universe. http://wwwphy. princeton.edu/ steinh/npr/. [Consultado: 12/01/2015]. (cursivas nuestras)

[7] Tegmark, M.: «Parallel Universes», en Barrow, J., P. Davies, P., Harper, C.: (eds.) Science and Ultimate Reality; quantum theory, cosmology, and complexity. Cambridge: Cambridge University Press, 2004, pp. 484, 486. (cursivas nuestras)

[8] Green, B.: La realidad oculta, cit., p. 417.

Thémata. Revista de Filosofía No53 (2016) pp.: 161-178. 
en viaje hacia el descubrimiento de las leyes finales» ${ }^{9}$. Leonard Susskind, otro proponente del multiverso, en un contexto de discusiones en torno a la demarcación científica, sentencia que la estructura de la ciencia con su método son autónomos y, por lo tanto, libres de cualquier dictamen que la filosofía pueda realizar; así dice: «La buena metodología científica no es un conjunto abstracto de reglas dictadas por los filósofos. Está condicionada y determinada por la propia ciencia y los científicos que crean la ciencia» ${ }^{10}$.

Desde estas instancias, parece ser que la ciencia se posiciona en una cima cognoscitiva suprema, capaz de explicarlo todo, habilitada para desbancar a cualquier otro saber que con diferentes recursos intenta contribuir a la explicación de la vasta y diversa realidad; y, de igual manera, capaz de cubrirse de cualquier influencia externa que le pretenda delimitar. De esta forma, la ciencia quedaría establecida como una empresa perfecta, sin límites y como la autoridad absoluta en cuanto a la descripción y explicación de la realidad.

Desde luego que estas actitudes ideológicas han sido criticadas desde la argumentación filosófica misma, con señalamientos a las inconsistencias y a la imposibilidad de tal intento utópico por instaurar un saber de tales magnitudes. La filosofía de Nicholas Rescher es uno de esos esfuerzos que se han dedicado a esta labor crítica, y por medio de ella, ofrecer una visión coherente y ponderada de la ciencia, en este caso de la cosmología física y sus límites.

\section{Imposibilidad de la ciencia perfecta: los límites de la teorización en la ciencia}

La ciencia puede ser entendida como la apropiación de la realidad natural y social por parte del ser humano, lo que a su vez implica una actividad cognoscitiva y práctica; o dicho de otro modo, como el control teórico y tecnológico del entorno, que juntamente se puede traducir en conocimiento y manipulación humana de lo real.

Desde estos presupuestos, ciencia sería "todo sistema de representación cognoscitiva y de control de la realidad ${ }^{11}$. Esta afirmación sitúa a

[9] Weinberg, S.: El sueño de una teoría final. Barcelona: Crítica, 2010, p. 191.

[10] Susskind, L.: El paisaje cósmico. Teoría de cuerdas y el mito del diseño inteligente. Barcelona: Critica, 2007, pp. 226-227. Para un estudio histórico de este tema, véase del mismo autor del presente artículo: Mayo, David. «Especulaciones y controversias en la cosmología física contemporánea: el problema de la creación en la hipótesis del multiverso, un análisis histórico». Próximo a publicar en la RCFC, volumen XVI, número 33, 2016, julio-diciembre.

[11] Solís, C., Sellés, M.: Historia de la Ciencia. Barcelona: Espasa, 2013, p. 19.

Thémata. Revista de Filosofía $\mathrm{N}^{\circ} 53$ (2016) pp.: 161-178. 
la ciencia - principalmente a las llamadas ciencias naturales o duras - en una clara posición determinante para la existencia humana, ya que el logro de hacer de la realidad natural algo comprensible y controlable para el ser humano, la constituye en el paradigma del saber, de la racionalidad y del progreso. Y esto para algunos, la convierte en la empresa cognoscitiva perfecta que puede dar razón de todo (lo que pueda ser explicable). Pero, según la filosofía de Nicholas Rescher, la ciencia no está próxima a ser un constructo perfecto y sin límites, su propia estructura hace imposible una labor racionalizadora en favor de modelos explicativos de la realidad total. La ciencia, recalca Rescher, es solo un saber entre varios, una clase de conocimiento o un proyecto epistémico — el más fiable y seguro ciertamente-, que no lo puede abarcar todo y que, por consiguiente, no lo puede explicar todo; pero que, al mismo tiempo, esta condición no es un defecto sino más bien una exigencia correspondiente a su cualidad de saber particular y específico. Es por eso que el filósofo de origen alemán sostiene, en consecuencia, que la realidad no se agota con la indagación científica, por cuanto, la explicación definitivamente verdadera de la realidad no es alcanzable, teniendo en cuenta la mencionada especificidad y limitación de los recursos metodológicos que la empresa científica requiere para elaborar su conocimiento. Así que, una perfección teórica de la ciencia iría más allá de las posibilidades metodológico-racionales fundamentales que le corresponden en su misión epistemológica objetiva de describir, explicar, predecir y controlar el orden natural regido por leyes.

En la realidad hay, afirma Rescher, más "que lo contemplado por la ciencia» ${ }^{12}$, es decir, que la realidad comprende y contiene cosas, fenómenos, problemas, etc., de mucha profundidad y complejidad cognitiva, lo que deja a la ciencia con evidencias incompletas e informaciones parciales ${ }^{13}$. Roger Trigg hace notar que: «Si el mundo contiene una sutil interacción entre el orden y el azar, de indeterminación y aleatoriedad, incluso entre las causas y la libertad, la ciencia tendrá un amplio espacio para el descubrimiento de la naturaleza de las cosas» ${ }^{14}$. Por tal motivo, la teorización científica desde su marco explicativo no lograría abarcar de manera exhaustiva la totalidad de lo real. De manera que las producciones científicas acerca de la realidad natural siendo correctas nunca serán definitivas y perfectas;

[12] Rescher, N.: Razón y valores en la era científico-tecnológica. Barcelona: Paidós, 1999, p. 113.

[13] Aquí Rescher se refiere, por ejemplo, al aspecto particular-experiencial de lo real el cual siempre exhibirá características que eluden la captación explicativa de la ciencia; también, a la esfera de los valores éticos y estéticos, y principalmente, a la «cuestiones últimas» de tipo metafísico.

[14] Trigg, R.: Rationality and Science: Can Science Explain Everything? Oxford: Blackwell Publishers, 1993, p. 193.

Thémata. Revista de Filosofía No53 (2016) pp.: 161-178. 
eso quiere decir que los modelos explicativos siempre serán falibles y corregibles, y, por lo tanto, no representarían, insistimos, la plena verdad de las $\operatorname{cosas}^{15}$. Rescher es enfático en señalar que el realismo científico que promueve una presentación de la verdad real del mundo, o en otros términos, una descripción exacta del mundo $-\mathrm{y}$ que con ello satisfacer los ideales cognitivos de unidad, consistencia, completud, definitud-, no es viable. Esto, a su vez, está sustentado en la idealización de la ciencia perfecta, idealización que tiene la sola función regulativa de impulsar el mejoramiento en la investigación. Así argumenta Rescher:

La historia de la ciencia muestra que nuestros 'descubrimientos' garantizados por el coherentismo inductivo del método científico constantemente requieren ajuste, corrección, reemplazo. No podemos decir que nuestras preguntas inductivas sobre cómo funcionan las cosas en el mundo que nos provean de la verdad (definitiva) real [...] La ciencia perfecta es una idealización —al igual que el realismo científico que viene automáticamente a su paso. [Esta idealización representa] la legitimidad de nuestros ideales cognitivos como reguladores inherentes en su utilidad como guías de consulta, y, en concreto, en su capacidad para guiar nuestros pensamientos y esfuerzos en direcciones constructivas y productivas. ${ }^{16}$

Así pues, la ciencia quedaría constituida como una empresa imperfecta. Su intento de lograr una comprensión definitiva de la naturaleza o, en este caso, una comprensión absoluta del cosmos, no es posible. Según Rescher, para que la ciencia pueda alcanzar el ideal de perfección debe cumplir con ciertas condiciones cognitivo-teóricas y activo-prácticas, esto es, conseguir la completitud teórica, informativa y de control del entorno.

En el área que nos interesa, la teorética, Rescher señala principalmente dos completitudes o acabamientos imprescindibles: la erotética y la predictiva. La erotética tiene que ver con la exigencia de dar respuestas «a todas las preguntas descriptivas y explicativas a que ella [la ciencia] se enfrenta como legítimamente planteables, y debe explicar todo lo que considera explicable» ${ }^{17}$. Ante esto, el filósofo afirma que en apariencia la ciencia podría estar dando respuesta a todas las preguntas que suscita el carácter problemático de la realidad — como en apariencia sucede con la cosmología especulativa-, pero con toda seguridad tales respuestas podrían resultar inadecuadas. Indudablemente, esta actitud colocaría a la

[15] Esta idea se acerca al concepto de verosimilitud popperiano, el cual sostiene que la verdad que es exigida para las teorías y que es colocada como el criterio de garantía para diferenciar las teorías verdaderas de las falsas o de la simple creencia, nunca es poseída de manera total, sino simplemente lo que se puede lograr son aproximaciones a tal verdad.

[16] Rescher, N.: Scientific Realism: A Critical Reappraisal. Dordrecht: Reidel Publishing, 1987, pp. 27, 31. (cursivas nuestras)

[17] Rescher, N.: Los límites de la ciencia. Madrid: Tecnos, 1994, p. 164.

Thémata. Revista de Filosofía $\mathrm{N}^{\circ} 53$ (2016) pp.: 161-178. 
ciencia fuera del aspecto estrictamente teórico-objetivo, para situarla en el ámbito ideológico y puramente especulativo, en el que el científico — en su pretensión omniabarcante - asume un cientificismo que deforma, distorsiona a la ciencia, "arrojando el manto de su autoridad sobre cuestiones a las que nunca pretendió atender» ${ }^{18}$. En contra de tal posición -ideológica-, la historia y el contexto actual de la ciencia cosmológica muestran y confirman que el alcance de nuestros conocimientos es lo suficientemente restringido para lograr la mencionada completitud. Más aún, para hacer asequible esa realización — de una ciencia de la naturaleza teoréticamente completa - sería necesario "ofrecer una teoría unificada completa y consistente de la interacción física que describiera todas las observaciones posibles» ${ }^{19}$. Para Rescher, el problema con dicha teoría radica en la imposibilidad de obtener un conocimiento absolutamente preciso de todas las interacciones físicas existentes y posibles, porque para ello se necesitaría - aparte de un conocimiento absoluto - un conocimiento o una teoría previa que garantice o controle que la plena adecuación y exactitud de todas las leyes, principios, observaciones, etc., se han obtenido. Pero eso no es posible, dado que tal teoría siendo última o final no podría, a pesar de todo, lógicamente auto-explicarse o auto-controlarse ${ }^{20}$; así afirma:

[...] para tener garantizado que el uso del estado actual de la física es una buena base para responder a esta cuestión, tendríamos que asegurarnos antes de que su visión de tales posibilidades es correcta; y en consecuencia, tener ya preestablecida su completitud. La idea de una completitud erotética consolidada naufraga ante la imposibilidad de encontrar una manera significativa de controlar su consecución. ${ }^{21}$

De igual forma, y como parte fundamental de la metodología científica, Rescher apunta que la inducción exigida por la teorización científica

[18] Rescher, N.: Razón y valores en la era científico-tecnológica, cit., p. 112.

[19] Citado por Rescher, en Rescher, N.: Los límites de la ciencia, cit., p. 168.

[20] La imposibilidad de la auto-explicación implica que una determinada teoría tendría que poseer externa e internamente y al mismo tiempo los elementos que le explican, lo cual, evidentemente, es lógicamente inconsistente. Nicholas Rescher hace una crítica a la llamada Teoría Final, Teoría del Todo (TOE) o teorías de la gran unificación (GUT) —que son aquellas que darían razón de todas las leyes y constantes del Universo, de su origen, de su estructura general, etc., asimismo, las que unificarían las cuatro fuerzas o interacciones fundamentales de la naturaleza-, aduciendo que existen varias limitaciones para el establecimiento de tal teoría, como por ejemplo, que ésta tendría que superar el elemento de la Integralidad, la finalidad, el punto muerto, los cuales estarían condesados en la imposibilidad de superar la no-circularidad explicativa. Véase en Rescher, N.: Nature and Understanding. The Metaphysics and Method of Science. Oxford: Clarendon Press, 2000. pp. 73-91. Véase también, por ejemplo, Weinberg, S.: El sueño de una teoría final. Barcelona: Crítica, 2010. Evans, J.: Watts, G.: Unification of Fundamental Forces. Cambridge: Cambridge University Press, 1990. Hawking, S.: The Theory of Everything. Beverly Hills: Phoenix Books, 2007.

[21] Rescher, N.: Razón y valores, cit., p. 140.

Thémata. Revista de Filosofía No53 (2016) pp.: 161-178. 
nunca podrá determinar las leyes de la naturaleza; en todo caso, los datos extraídos de las observaciones solo podrían falsar las teorías. Con todo, la confirmación empírica es imprescindible para el quehacer científico, de lo contrario no sería propiamente ciencia y ella misma podría decir cualquier cosa de un carácter epistemológicamente distinto y, eventualmente, desacertado. Rescher advierte que: «Uno no puede estar seguro de poner el punto final, si [la ciencia] se basa en consideraciones puramente teóricas $»^{22}$. Por esa razón, la inducción se constituye en un elemento fundamental en el proceso de teorización científica, dado que a partir de las observaciones la generalización inductiva provee de un patrón general de las regularidades manifestadas por la naturaleza, para luego incluirlas en el espectro de construcciones teóricas, todo con el fin de dar respuesta a las preguntas correspondientes al ámbito natural o empírico. Este requerimiento inductivo, además de indicar que la referencia a los datos observacionales es un requisito indispensable, es ante todo una limitante para el perfeccionamiento científico, considerando que a medida que la exploración empírica avanza nuevos datos van corrigiendo y enriqueciendo las concepciones científicas vigentes. Esto quiere decir que la indagación científica siempre estará sujeta a cambios e imprecisiones, a causa del problema del carácter contingente de la realidad natural. Este último problema está más presente en la exigencia de completitud predictiva, la cual analizaremos en el siguiente punto junto al tema de la explicación científica.

Un presupuesto implícito de la racionalidad científica que es necesario destacar es: que los límites en la teorización son un indicativo de racionalidad; ésta exige delimitar los ámbitos, los recursos, los métodos y, por tanto, las respuestas, a fin de hacer lo mejor posible (optimizar) a partir de las razones más poderosas. La racionalidad restringe el ímpetu de la ciencia $-\mathrm{y}$ de cualquier otro ámbito humano- por querer abarcarlo todo, por querer solucionar problemas demasiado complejos para los cuales no está capacitada o no posee los medios necesarios; y esto es lo que la convierte en una empresa cognitiva competente a diferencia de la pseudociencia o el saber vulgar, en los que, según Rescher, "todo encaja demasiado bien en su sitio» ${ }^{23}$. En efecto, la racionalidad le prohíbe a la ciencia hacer una cobertura total de la realidad, incluir o adoptar para sí cualquier idea o mecanismo, puesto que la consecuencia inmediata a esto sería la desorganización de su estructura interna y, como resultado, la imposibilidad de realizar el control racional de la realidad natural.

Dentro de las demandas que la racionalidad le imprime a la ciencia está lo que Rescher llama la sistematización del mecanismo operativo de

[22] Rescher, N.: Los límites de la ciencia, cit., p. 169.

[23] Ibídem, p. 138.

Thémata. Revista de Filosofía №53 (2016) pp.: 161-178. 
la ciencia, que es la que determina la optimización de sus objetivos cognoscitivos. La razón de esto, se debe a que la validez cognoscitivo-teórica de la ciencia requiere de una definición y una ordenación de los aspectos descriptivos que hacen posible la investigación racional, aportando los criterios, las normas y los patrones para la aceptabilidad y adecuación al proceso racional, los cuales, a su vez, están orientados a la verdad presuntiva y a la corrección fáctica. Por ello, uno de los principios fundamentales que determinan y especifican la racionalidad cognoscitiva es «la búsqueda de la verdad y el logro de respuestas correctas» ${ }^{24}$. Es así como la obtención de verdades genuinas - pero nunca acabadas - se constituye como el objetivo de la sistematización; ésta procura poner en orden todos los datos disponibles, sean estos los datos de los sentidos, verdades o teorías previas (elementos experienciales o discursivos), o también los datos inciertos o poco claros. Todo esto con el fin de aceptar como verdades sólo aquellas que son coherentes entre sí. Esta es la denominada teoría coherentista de la verdad que el filósofo de Pittsburgh propone con el propósito de lograr el paso "de la incoherencia a la coherencia, del desorden al sistema, de candidatos a verdad a verdades calificadas» ${ }^{25}$.

Este ideal regulativo de la coherencia de la verdad pasa a constituirse en el soporte que permitirá superar el problema entre la apariencia y la realidad, entre afirmaciones hipotéticas o subjetivas y las afirmaciones ontológicamente objetivas en el nivel del ser o la realidad. De ahí que uno de los componentes esenciales del criterio coherentista de la verdad sea la adecuación a la facticidad. Por ese motivo, la verdad fáctica, constituida a partir del mundo real, es indispensable en cualquier teoría o declaración científica, pues sólo de esta manera se podrán aceptar como verdaderas. Lo ontológicamente real y objetivo es condición necesaria para una descripción y explicación correcta y verdadera del mundo — por supuesto siempre provisional y tentativa—; y esta cualidad — de lo ontológicamente real— tiene una clara identificación en lo operativo, en la manifestación de actividad intrínseca y extrínseca de todas las cosas; esto por cuanto:

Ser real es estar en condiciones de provocar un impacto en algún lugar sobre algo, de tal manera que un equipo adecuado, lo dotado de mente, una criatura inteligente puedan detectarlo. Lo que es real en el mundo debe provocarle a éste algún cambio, que es, en principio, detectable. ${ }^{26}$

[24] Rescher, N.: La racionalidad. Una indagación filosófica sobre la naturaleza y la justificación de la razón. Madrid: Tecnos, 1993, p. 183.

[25] Rescher, N.: Sistematización cognoscitiva. México: Siglo XXI, 1981, p. 85.

[26] Rescher, N.: Nature and Understanding. The Metaphysics and Method of Science. Oxford: Clarendon Press, 2000, pp. 121-122.

Thémata. Revista de Filosofía No53 (2016) pp.: 161-178. 


\section{Límites racionales de la explicación y la predicción científica}

La racionalidad que caracteriza a la ciencia está enraizada dentro de sus mismos objetivos como quehacer teorético. Dichos objetivos se condensan en el conocimiento de leyes que son las que estructuran nuestra comprensión de los sucesos del pasado y conducen a la previsión del futuro, y esta labor recae sobre la explicación y la predicción ${ }^{27}$. Estas, asimismo, representan un control sobre la naturaleza el cual es tomado como criterio de perfección. Pero la predicción, como parte de la completitud para la perfección de la ciencia, se enfrenta con varias limitaciones que le impiden a la ésta tener un control absoluto sobre el conocimiento del acaecer o no de los sucesos. De acuerdo con Rescher, el control cognitivo que representa la predicción se circunscribe a condicionamientos epistemológicos y ontológicos, los cuales son el impedimento para que la ciencia no pueda ofrecer resultados con absoluta precisión y certeza.

En cuanto a los límites ontológicos, el filósofo considera que las cualidades que posee la realidad natural hacen que el futuro sea indeterminado, abierto; esto es, que los sucesos estocásticos o azarosos producen un desconocimiento de las condiciones iniciales e inexactitud de la información nomológica, lo que conduce a la imposibilidad de hacer inferencias certeras y de ofrecer valores absolutamente precisos para los fenómenos ulteriores ${ }^{28}$. De modo que con la ausencia de conocimiento preciso de las condiciones iniciales y de la inexactitud de los valores de las regularidades de la naturaleza, la ciencia moderna se ha visto en la obligación de utilizar la teoría de la probabilidad para realizar las inferencias a los sucesos aleatorios; es decir, que en ciertos casos la distribución de probabilidades se convierte en la predicción científica. Y esto, seguramente, no es ausencia de la predicción en sí misma sino un fallo en la certeza de la predicción. Considerando esto, se tiene que hay aspectos de la realidad misma, a saber, la indeterminación y la contingencia, que le impiden a la ciencia poseer una predicción racional completa; y aunque el futuro esté prefigu-

[27] A este respecto Wenceslao González señala que: «La predicción incide en la Ciencia Básica orientada a la ampliación del conocimiento [...] La predicción es también un test que sirve para dirimir si un enunciado es o no científico, lo que permite evaluar teorías científicas.» En González, W. J.: La predicción científica. Concepciones filosófico-metodológicas desde H. Reinchenbach a N. Rescher. Barcelona: Montesinos, 2010, p. 11.

[28] El indeterminismo ha llegado a ser el carácter primordial de la realidad física, y a partir de él, el predominio de las interpretaciones probabilísticas que buscan explicar el carácter estadístico, por ejemplo, de la teoría cuántica. Evandro Agazzi habla acerca de la discrepancia entre continuidad y discontinuidad en el mundo microfísico; la conceptualización de masa y sus dimensiones espacio-temporales; el cambio del determinismo al probabilismo en las leyes naturales, y la reconsideración del principio de causalidad. Véase Agazzi, E.: La ciencia y el alma de Occidente. Madrid: Tecnos, 2001, pp. 183ss.

Thémata. Revista de Filosofía №53 (2016) pp.: 161-178. 
rado en las leyes de la naturaleza, su esencia per se está sustentada en la contingencia. En palabras de Rescher: «Este aspecto de la realidad [la prefiguración del futuro en las leyes naturales] es crucial para la predicción racional [...] Pero el futuro aún no ha entrado en escena; no existe, por lo menos no todavía» ${ }^{29}$.

Por su parte, los límites epistemológicos están en conexión con lo indicado anteriormente sobre el futuro, al ser éste abierto resulta cognoscitivamente inaccesible; es "un modelo de naturaleza que implica que cierta clase de cosas son incognoscibles» ${ }^{30}$. Este carácter imprevisible - contingente e indeterminado- de la naturaleza impide al científico obtener ciertos datos cruciales para así lograr la predicción racional perfecta. Por esa razón, solamente puede realizarse una anticipación conjetural a partir de una configuración inferencial de la información disponible. Esta información — cuyo fin es la predicción de un futuro abierto - está contenida en un pasado y un presente que son comprendidos a partir de ciertos patrones de estabilidad (pattern stability), de la disponibilidad de datos (data availability), y de patrones de discernibilidad (pattern discernibility). Simultáneamente, estos patrones deben estar ajustados entre sí, considerando que son las «tres precondiciones indispensables para la predicción racional» ${ }^{31}$. Estos patrones se resumen de la siguiente manera:

1) Que la información relevante acerca del pasado-y-presente pueda ser obtenida adecuadamente de una manera exacta y fiable. 2) Que el conjunto de datos exhiba patrones discernibles; 3) En la medida en que los patrones expuestos son estables, así como esta característica estructural manifiesta una consistencia que también continúe en el futuro. ${ }^{32}$

Todo esto significa que la predicción racional descansa en la eficiencia del ajuste de patrones, el que, asimismo, gira en torno a un vínculo entre los datos obtenidos y las afirmaciones predictivas hechas por los expertos. Para ello debe darse una justificación racional que garantice la exactitud de los principios inferenciales que soportan las predicciones; pero tal justificación no es posible en la práctica, sino únicamente de una manera irremediablemente restringida. Estas son las deducciones de Rescher:

[29] Rescher, N.: Predicting the Future. An Introduction to the Theory of Forecasting. New York: State University of New York Press, 1998, p. 70.

[30] Rescher, N.: Los límites de la ciencia, cit., p. 175.

[31] Rescher, N.: Predicting the Future, cit., p. 86.

[32] Ídem.

Thémata. Revista de Filosofía №53 (2016) pp.: 161-178. 
las perspectivas de la predicción de la ciencia están limitadas por el papel del azar y el caos en el esquema del mundo de las cosas, de las cuales la ciencia natural misma nos informa. Un Universo natural —como el nuestro- el cual está localizado en la frontera turbia entre la regularidad y el desorden, entre la uniformidad y la variedad, entre la elegante simplicidad y la intratable complejidad [...] En tal ámbito, la predicción, aunque no es factible, será una propuesta viable sólo a un grado bastante restringido. ${ }^{33}$

Con respecto a la explicación científica, lo que le atribuye cientificidad son básicamente dos componentes, a saber, la «materia o el contenido observacional (sucesos particulares-concretos), y el método utilizado (la subsunción de los sucesos bajo leyes confirmadas y contrastadas)» ${ }^{34}$. En relación a este último, las leyes de la naturaleza son el recurso o la entrada (input) necesaria que el formato de la explicación científica requiere para dar inteligibilidad al suceder de los eventos naturales. Es con el factor de legalidad (lawfulness) que se proporciona una garantía racional que es la que le imputa inevitabilidad al acontecer de los fenómenos, y esto en conformidad con los sistemas teóricos; "consiste en evidencia observacional y experimental para la generalización, y esto combinado con la capacidad de la generalización para adherirse con otros enunciados en una estructura teorética (un sistema axiomático científico)» ${ }^{35}$. Este es el aspecto modal que el conocimiento científico demanda para ofrecer sus productos cognoscitivos seguros — continúa Rescher-, lo que significa dar una respuesta a ¿cómo las cosas operan?, «¿Cuál es el caso y ¿Por qué este es el caso?»36. Así, el mecanismo científico de la explicación naturalista legitima el devenir, el desarrollo o la actividad de un concreto estado de cosas mediante la cobertura de las leyes de la naturaleza. Sobre eso se sostiene que en toda explicación científica deben estar presentes estos requisitos: datos empíricos (datos observacionales y/o experimentales) y las leyes de la naturaleza (generalizaciones incluidas en sistemas teóricos); y forzosamente, estos elementos son parte de la cuestión cuando de lo que se quiere dar cuenta es de la existencia y la estructura general del Universo. Rescher lo expone del siguiente modo:

[33] Rescher, N.: Predicting the Future, cit., pp. 173-174.

[34] Rescher, N.: Scientific Explanation. New York: The Free Press, 1970, p. 10.

[35] Temple, D.: «Can science know what's necessary?» en Rescher, N. (ed.): The limits of lawfulness. Studies on the scope and nature of scientific knowledge. Boston: University Press of America, 1983. pp. 43-50.

[36] Ibídem, p. 13. (cursivas del autor)

Thémata. Revista de Filosofía $\mathrm{N}^{\circ} 53$ (2016) pp.: 161-178. 
Para las explicaciones científicas, por su propia constitución como tales, deben hacer uso de las leyes de la naturaleza en su razonamiento. Pero esta estrategia es simplemente inasequible en el presente caso [de explicaciones últimas]. Pues esas leyes de la naturaleza requeridas para la explicación científica son en sí mismas una parte —una parte de esencial y fundamental — de la constitución de la realidad física. Y son, por lo tanto, una parte del problema y no instrumentalidades disponibles para su resolución. ${ }^{37}$

\section{El horizonte de inteligibilidad de la cosmología física}

La explicación puede ser considerada — desde la perspectiva rescheriana- como la tarea principal de la ciencia, ya que esta está presente en cierto modo en todos sus demás objetivos. Y siendo la explicación medular en los objetivos de la ciencia y su poder demostrativo el más seguro — por ser objetivo y racional—, la pregunta qué es necesario responder es, ¿a qué no puede dar respuesta la ciencia? o ¿Qué queda por fuera de la competencia de la ciencia? Rescher afirma que en principio nada de lo explicable queda por fuera de su competencia. No obstante, todos los fenómenos, propiedades, estado de cosas, sucesos o todas las facetas de lo que sucede en el mundo, deben ser subsumidos en un marco explicativo pertinente, es decir, en un grupo de conceptos y principios establecidos por la materia y el método. Juntamente a esto, hay también alguna clase de ámbitos o realidades que están localizados fuera del alcance de la ciencia, en la medida en que se sitúan más allá de sus límites operativos. Estos límites de la explicación científica son establecidos a partir de los principios últimos o axiomas, que son el término del camino de la estructura explicativa causal-nomológica. La argumentación se articula como sigue:

las leyes se explican por derivación de otras leyes. Éste es, claramente, un proceso que debe alcanzar un tope. El conjunto de las leyes de la naturaleza - hasta donde seamos capaces de desarrollarlo- es siempre finito. [...] la explicación subjuntiva de las leyes debe llegar finalmente 'al término del camino'. Aquí alcanzamos siempre ciertos principios últimos, 'axiomáticos'.

Estos principios últimos de la explicación están vinculados a las «preguntas últimas» que corresponden a sucesos de carácter general o primordial, considerados tradicionalmente como de tipo metafísico; para lo cual Rescher menciona algunas de las siguientes: «ipor qué hay algo más bien que nada? ¿Por qué hay cosas físicas? ¿Por qué existe cosa alguna? ¿Por qué es la naturaleza un cosmos ordenado? ¿Por qué hay leyes (uniformidades, regularidades) naturales?» ${ }^{38}$. Las respuestas a tales cuestionamientos último-metafísicos pueden venir de diferentes marcos explica-

[37] Rescher, N.: On Explaining Existence. Frankfurt: Ontos, 2012, p. 127.

[38] Ibídem, p. 148.

Thémata. Revista de Filosofía №53 (2016) pp.: 161-178. 
tivos tales como: «la justificación teológica que postula a un dios-creador, la justificación mística que invoca un 'azar' misterioso, la justificación naturalista que mira hacia alguna exigencia interna del Universo en sí mismo» ${ }^{39}$. Para Rescher, ante este tipo de preguntas no se puede establecer una explicación científica racional y coherente debido a que no es posible, en primera instancia, aplicar el método científico de subsunción de sucesos no-existentes a leyes naturales también inexistentes. Esto significa la ausencia del material empírico y la inaplicabilidad, por tanto, del método, que son necesarios para completar la explicación científica. Por esa razón, y en referencia propiamente al tema de la hipótesis cosmológica del multiverso, el pensador germano-americano afirma:

si estos otros mundos están verdaderamente separados de nosotros — si ellos no interactúan con nosotros de la manera en que su innovación induce necesariamente para explicar las características de nuestro mundo- entonces nosotros cortamos no solo físicamente, sino también con el contenido probatorio de todos esos mundos, y no tenemos ninguna razón real para aceptarlos. ${ }^{40}$

En todo caso, si tal explicación última llega a darse, la misma ya no sería científica, pues la racionalización que realiza el método científico impide la explicación desde «estadios previos en función de estados posteriores» ${ }^{41}$, en tanto que estados previos no empíricos. De manera que, una explicación causal-eficiente desde leyes y condiciones iniciales específicas para los orígenes de la totalidad del espacio-tiempo que conforman nuestro Universo visible, o bien, la propuesta de una causa última física, es inconsistente con la capacidad racional de la ciencia. De otro modo, la ciencia se reduciría a una pura especulación metafísica, para lo cual no cuenta con las herramientas conceptuales ni metodológicas apropiadas. Por lo tanto, el límite del poder explicativo de la ciencia debe ubicarse dentro de los límites del dominio inteligible del Universo (tetradimensional), este es, el dominio en el que todo suceso o fenómeno puede ser percibido y entendi-

[39] Rescher, N.: Scientific Explanation, cit., p. 140.

[40] Rescher, N.: Epistemological Studies. Frankfurt: Ontos, 2009, p. 95. (cursivas nuestras). Dentro de los temas de la cosmología especulativa la hipótesis cosmológica del multiverso es considerada como la más especulativa y extravagante a la vez, y la que más atención atrae no solamente a la comunidad científica, y esto no solamente por sus contenidos sino también por los insinuantes cambios epistemológicos que conlleva, los cuales se distancian de las teorías pertenecientes al modelo estándar. Así advierte el historiador de la ciencia Helge Kragh: «El multiverso... es probablemente el más radical de los intentos modernos para establecer la ciencia fundamental sobre nuevas bases. En efecto, términos tales como `revolución' y 'cambio de paradigma' son comúnmente escuchados en la controversia sobre el multiverso». En Kragh, H.: Higher speculations, cit., p. 363.

[41] Rescher, N.: Scientific Explanation, cit., p. 156.

Thémata. Revista de Filosofía №53 (2016) pp.: 161-178. 
do por el aparato conceptual, discursivo y metodológico de la ciencia. A este respecto, Milton Munitz recalca que la comprensión a lo que hace referencia el concepto «el Universo» no se obtiene hasta que no especifiquemos ciertas características en un modelo cosmológico, tales como: «que ha sido sometido a un proceso de recolección de datos observacionales, la identificación de distintos tipos de objetos y procesos, la aplicación de leyes aceptadas para la descripción, explicación, predicción, y retrodicción específica de eventos e incidentes, la realización de cálculos, y, finalmente, la contrastación de sus inferencias ${ }^{42}$. Significa esto que la comprensión - científica - de lo que es el Universo (o un universo) está necesariamente condicionada por aspectos observacionales y conceptuales en consonancia con los procedimientos científicos. En resolución, no podemos dar razón de nuestro Universo conocido utilizando como explicación causal a otros universos (o al multiverso) extra-dimensionales, desconocidos e incomprensibles para nuestro razonamiento científico. Así concluye Rescher:

si el suceso a explicar es 'el origen de la existencia en su totalidad', no podemos proporcionar una explicación de él en términos del impacto causal de sucesos antecedentes. Pues no hay (ex hypothesi) suceso antecedente alguno... [Esto] No hace otra cosa que establecer lo absurdo que es pedir que se provea a un suceso originario de una explicación específicamente causal. Está claro que no tiene sentido pedirle a la ciencia lo que es imposible por principio. ${ }^{43}$

\section{Conclusión}

Efectivamente, la pretensión científica - llevada a cabo por la cosmología física-, de ofrecer respuestas o explicaciones a sucesos inequívocamente últimos correspondientes a la especulación metafísica, tropieza con serios obstáculos filosóficos; impedimentos cimentados en una racionalidad delimitada por un modelo metodológico definido, esto desde el límite estructural de la propia ciencia. Con ello, el quehacer científico, como ha mostrado Nicholas Rescher, está muy lejos de ser una empresa ilimitada, omniabarcante y perfecta. El filósofo deja claro que son la misma realidad natural y la propia constitución epistemológica de la ciencia, las que restringen la validez de los esfuerzos cosmológicos contemporáneos por ofrecer explicaciones coherentes para el origen y para la razón de ser del ajuste preciso de las leyes y constantes que hacen posible la evolución del Universo. En vista de ello, las especulaciones cosmológicas de la ciencia,

[42] Munitz, M. K.: Cosmic Understanding: Philosophy and Science of the Universe. Princeton: Princeton University Press, 1986, p. 176.

[43] Rescher, N.: Los límites de la ciencia, cit., p. 26. (cursivas nuestras)

Thémata. Revista de Filosofía №53 (2016) pp.: 161-178. 
como en el caso de las hipótesis del multiverso, no pasan de ser metafísica de muy dudosa consistencia discursiva y precisión conceptual. Finalmente, advertimos que la ciencia se encuentra necesariamente con la filosofía en su intento por comprender la realidad en su totalidad o a un nivel fundamental, y que aquella misma requiere de esta para comprender su propia racionalidad.

\section{Bibliografía}

\section{Obras de Nicholas Rescher}

Rescher, N.: Epistemological Studies. Frankfurt: Ontos, 2009.

Rescher, N.: Nature and Understanding. The Metaphysics and Method of Science. Oxford: Clarendon Press, 2000.

Rescher, N.: Razón y valores en la era científico-tecnológica. Barcelona: Paidós, 1999.

Rescher, N.: Predicting the Future. An Introduction to the Theory of Forecasting. Albany: State University of New York Press, 1998.

Rescher, N.: Los límites de la ciencia. Madrid: Tecnos, 1994.

Rescher, N.: La racionalidad. Una indagación filosófica sobre la naturaleza y justificación de la razón. Madrid: Tecnos, 1993.

Rescher, N., On Explaining Existence. Frankfurt: Ontos, 2012.

Rescher, N.: Scientific Realism: A Critical Reappraisal. Dordrecht: Reidel Publishing Co., 1987.

Rescher, N.: Sistematización cognoscitiva. México: Siglo XXI, 1981.

Rescher, N.: Scientific Explanation. New York: The Free Press, 1970.

\section{Bibliografía secundaria}

Barrow, J., P. Davies, P; C. Harper, C.: (eds.) Science and Ultimate Reality; quantum theory, cosmology, and complexity. Cambridge: Cambridge University Press, 2004.

Carr, B.: (ed.) Universe or Multiverse? Cambridge: Cambridge University Press, 2007.

Goenner, H.: «What kind of science is cosmology?» Annalen der Physik (Leipzig) 522, Nr. 6, 389-418 (2010); arXiv: astro-phy/2010.5532. pp. 1-62.

González, W. J.: La predicción científica. Concepciones filosóficometodológicas desde H. Reinchenbach a N. Rescher. Barcelona: Montesinos, 2010.

Green, B.: La realidad oculta. Universos paralelos y las profundas leyes del cosmos. Barcelona: Crítica, 2011.

Kragh, H.: Higher Speculations: Grand Theories and Failed Revolutions in Physics and Cosmology. New York: Oxford University Press, 2011. 
Linde, A.: «Infation, quantum cosmology, and the anthropic principle», en Barrow, J., P. Davies, P., Harper, C.: (eds.) Science and Ultimate Reality; quantum theory, cosmology, and complexity. Cambridge: Cambridge University Press, 2004, pp. 426-458.

Munitz, M. K.: Cosmic Understanding: Philosophy and Science of the Universe. Princeton: Princeton University Press, 1986.

Solís, C., Sellés, M.: Historia de la Ciencia. Barcelona: Espasa, 2013.

Steinhardt, P. J. [en línea]: «A Brief Introduction to the Ekpyrotic Universe», http://wwwphy.princeton.edu/ steinh/npr/ [Consultado: 12/01/2015].

Susskind, L.: El paisaje cósmico. Teoría de cuerdas y el mito del diseño inteligente. Barcelona: Critica, 2007.

Tegmark, M.: «Parallel Universes», en Barrow, J., P. Davies, P., Harper, C.: (eds.) Science and Ultimate Reality; quantum theory, cosmology, and complexity. Cambridge: Cambridge University Press, 2004, pp. 459-491.

Temple, D.: «Can science know what's necessary?» en Rescher, N. (ed.): The limits of lawfulness. Studies on the scope and nature of scientific knowledge. Boston: University Press of America, 1983. pp. 43-50.

Trigg, R.: Rationality and Science: Can Science Explain Everything? Oxford: Blackwell Publishers, 1993.

Weinberg, S.: El sueño de una teoría final. Barcelona: Crítica, 2010. 